\title{
Avaliação da capacidade funcional de idosos na Unidade Básica de Saúde da Familia São Geraldo, município de Volta Redonda, RJ
}

\section{Functional capacity evaluation of elderly person registered in a Family Health Program located at São Geraldo neighborhood, in the city of Volta Redonda, RJ}

\author{
1 Suzana Angélica Silva Lustosa \\ 2 Márcia Dorcelina Trindade Cardoso \\ 3 Gabriel Vassalo de Souza gabriel.vassalo@hotmail.com \\ 3 Ana Paula Campos de Souza \\ ${ }^{3}$ Alice Juliana Otoni Muller Santos
}

\footnotetext{
Discente do Centro Universitário de Volta Redonda, UniFOA.

Docente do Centro Universitário de Volta Redonda, UniFOA.

3 Graduação em Medicina pelo Centro Universitário de Volta Redonda, UniFOA.
}

\section{RESUMO}

O envelhecimento faz com que a independência funcional diminua, acarretando, consequentemente, em uma redução da qualidade de vida. A manutenção da capacidade funcional dos idosos é de fundamental importância para realização das atividades básicas e instrumentais da vida diária. 0 presente estudo possui como objetivo avaliar a capacidade funcional de idosos cadastrados no Programa de Saúde da Família, no bairro São Geraldo, no município de Volta Redonda, RJ. Para realizar essa avaliação, foi aplicado o questionário validado $\mathrm{HAQ}-20$ (Stanford Health Assessment Questionnaire), em sua versão brasileira, com uma amostra de $\mathrm{n}=40$ idosos, sendo estes, o total dos que frequentaram a unidade de saúde em um período estipulado previamente (setembro, 2012). Foram acrescentadas duas variáveis para determinação de gênero e idade e sua relação com a capacidade funcional encontrada. Foi encontrado um total de $\mathrm{n}$ $=22$ idosos do gênero masculino (55\%) e $n=18$ idosos do gênero feminino (45\%). Em relação à idade, os idosos foram divididos em quatro grupos, sendo de 60 a 70 anos, $\mathrm{n}=22$ idosos (55\%); 71 a 80 anos, $n=11$ idosos (27,5\%); entre 81 e 90 anos $\mathrm{n}=5$ idosos (12,5\%) e com mais de 90 anos, $\mathrm{n}=$ 2 idosos (5\%). Quase $80 \%$ dos participantes apresentaram uma boa capacidade funcional, sendo classificados em "sem incapacidade" ou com "alteração leve da capacidade funcional", sendo, portanto, capazes de realizarem de forma adequada as suas atividades do dia a dia.

\section{Palavras-chave}

Idosos; capacidade funcional; Programa de Saúde da Família.

\begin{abstract}
As chronological age increases, functional independence and quality of life reduces. It is very important to maintain functional capacity of elderly person, in order to preserve independency in essential activities. This research evaluates functional capacity of elderly person registered in a Family Health Program located at São Geraldo neighborhood, in the city of Volta Redonda - RJ, Brazil. A valid questionnaire (Stanford Health Assessment Questionnaire) has been used to evaluate this functional capacity. In total, 40 elderly people have been investigated in a stipulated period of time (September, 2012). Age and gender have been analyzed. Out of the 40 analysed, twentytwo $(55 \%)$ were men and eighteen (45\%) were woman. Fifty - five (22) percentage of the population analyzed aged 60 to $70,27,5 \%(11)$ aged 71 to $80,12,5 \%$ (5) aged 81 to 90 , and $5 \%$ (2) aged more than ninety. The results showed that almost $80 \%$ of the elderly people had a good functional capacity therefore they are capable of performing ordinary daily activities.
\end{abstract}

\section{Key words}

Elderly, functional capacity, Family Health Program.

\section{Como você deve citar?}

SOUZA, Gabriel Vassalo de et al. Avaliação da capacidade funcional de idosos na Unidade Básica de Saúde da Família São Geraldo, município de Volta Redonda, RJ. Cadernos UniFOA, Volta Redonda, n. 32, p. 91-98, dez., 2016. 


\section{INTRODUÇÃo}

O fato mais marcante para as sociedades atuais é o processo de envelhecimento populacional observado em todos os continentes (BRASIL 2006). Segundo Lacourt e Marini (2006), dentre as principais alterações que surgem com o avanço da idade, está o decréscimo da função muscular, que afeta diretamente a capacidade de realizar tarefas do dia a dia, diminuindo a independência funcional e, desse modo, interferindo na qualidade de vida do idoso. Assim, a capacidade funcional surge como um valor ideal para que o idoso possa viver de forma independente e autônoma, sendo capaz de realizar suas atividades físicas e mentais necessárias para manutenção de suas atividades básicas, como: tomar banho, vestir-se, realizar higiene pessoal, transferir-se, alimentar-se, manter a continência, preparar refeições, ter o controle financeiro, etc (ALENCAR et al., 2008).

A avaliação funcional pode ser compreendida como uma tentativa sistematizada de avaliar de forma objetiva os níveis de que uma pessoa está funcionando numa variedade de áreas, utilizando diferentes habilidades (BRASIL, 2009). Segundo Duarte, Andrade e Lebrão (2007), a avaliação funcional pode ser definida como uma tentativa de medir, de forma objetiva, os níveis de que uma pessoa é capaz de desempenhar atividades ou funções em diferentes áreas, utilizando-se de várias habilidades para o desempenho das tarefas da vida cotidiana, para a realização de interações sociais, em suas atividades de lazer e em outros comportamentos requeridos em seu dia a dia. Portanto, o presente estudo tem como objetivo avaliar a capacidade funcional de idosos na Unidade Básica de Saúde da Família São Geraldo, no município de Volta Redonda, RJ.

\section{MATERIAIS E MÉTODOS}

Existem inúmeras escalas que servem para quantificação da capacidade funcional em idosos, muitas delas validadas no Brasil, porém com limitações principalmente no que se refere à sua especificidade. Elas devem ser breves, simples e de fácil aplicação, para que atinjam o seu principal objetivo, que é servir como instrumento rápido de avaliação, triagem e estratificação de risco, para que possam ser aplicadas por pessoal de múltiplas formações profissionais e em qualquer unidade básica de saúde (COSTA et al., 2001). Nesta pesquisa, foi escolhido o questionário HAQ-20 (Stanford Health Assessment Questionnaire).

A entrevista foi dividida em duas partes. A primeira parte constava a determinação de gênero (masculino e feminino) e faixa etária (de 60 a 70 anos, de 71 a 80 anos, de 81 a 90 anos ou acima de 90 anos). A segunda parte constava o questionário HAQ-20, em sua versão brasileira, composto de 20 questões diretas, distribuídas em oito domínios: vestir-se, levantar-se, alimentar-se, caminhar, higiene pessoal, alcançar objetos, apreender objetos e outras atividades. Nesse questionário, a maior pontuação na soma dos valores absolutos de todos os domínios é de 60 pontos e a menor é de 0 (zero). A pontuação é obtida a partir da somatória das respostas de todos os domínios, sendo que a menor pontuação reflete a melhor capacidade funcional e quanto maior a pontuação, pior a capacidade funcional demonstrada. Já para quantificar a alteração da capacidade funcional, é calculada a mediana de todos os domínios do questionário, que varia de 0 (zero) a 3 (três), em que de $0-1$, o sujeito apresenta alteração leve da capacidade funcional ou não apresenta incapacidade funcional; de 1-2, alteração moderada e 2-3, alteração grave da capacidade funcional.

Como todo instrumento, o HAQ tem limitações e, geralmente, não captura a inabilidade associada com a disfunção sensorial do órgão ou a disfunção psiquiátrica e não mede diretamente a satisfação do paciente ou o trabalho em rede social. Contudo, tais variáveis ou outras do interesse do usuário podem prontamente ser adicionadas (BRUCE; FRIES, 2003). Realizou-se um estudo transversal, no qual foram entrevistados os idosos que frequentaram a unidade de saúde no mês de setembro de 2012, sendo o 
número amostral $n=40$ idosos. Os responsáveis pela Unidade de Saúde e toda a equipe foram esclarecidos sobre a natureza da pesquisa, seus objetivos e procedimentos.

Todos os participantes assinaram o Termo de Consentimento Livre e Esclarecido, concordando em participar do estudo. Todos os participantes tiveram a liberdade de suspender ou encerrar a participação no estudo a qualquer momento sem nenhum prejuízo. Após leitura e assinatura do TCLE (termo de consentimento livre e esclarecido), foi aplicado o questionário. Os resultados foram tabulados no programa Microsoft Excel e foram feitos gráficos para ilustrar e facilitar o entendimento.

A pesquisa de campo foi iniciada após aprovação pelo Comitê de ética e pesquisa do Hospital Municipal Dr. Munir Rafful - Volta Redonda, respeitando-se o cumprimento dos princípios éticos contidos na Declaração de Helsinki (1964, reformulada em 1975, 1983, 1989, 1996 e 2000), da World Medical Association (http://www.wma.net/e/policy/b3.htm).

\section{CRITÉRIOS DE INCLUSÃO}

Pessoas com idade igual ou superior a 60 anos, de ambos os sexos, com condições de responder aos instrumentos, estarem cadastradas na Estratégia de Saúde da Família São Geraldo, do município de Volta Redonda - RJ, no período da coleta de dados e aceitarem participar da pesquisa.

\section{CRITÉRIOS DE EXCLUSÃO}

Estar acamado, apresentar doenças degenerativas, em coma e/ou com comprometimento cognitivo, de audição e de comunicação.

\section{RESULTADOS E DISCUSSÃO}

Como citado anteriormente, o número amostral foi de $n=40$ idosos, todos cadastrados na estratégia de saúde da família do bairro São Geraldo, do município de Volta Redonda, interior do Estado do Rio de Janeiro. Em relação ao gênero (Gráfico 1), $n=22$ idosos (55\%) eram do sexo masculino e $n$ $=18$ idosos (45\%) do sexo feminino, o que diverge da maioria dos estudos com a população idosa, em que o gênero feminino encontra-se, normalmente, em maior número, quando relacionado ao gênero oposto. Isso pode ter relação com o maior índice de mortalidade do sexo masculino na terceira idade. Para Veras (1994), as mulheres vivem mais, pois são menos expostas a riscos, acidentes domésticos e de trabalho, acidentes de trânsito, homicídios, suicídios, consomem menos tabaco e álcool, fazem uso mais frequente dos serviços de saúde. Entretanto, não são apenas esses fatores que justificam a longevidade nas mulheres: existem os fatores biológicos e genéticos que, embora ainda não estejam plenamente esclarecidos, contribuem para o prolongamento de suas vidas. 
Gráfico 1 - Distribuição entre gênero masculino e feminino

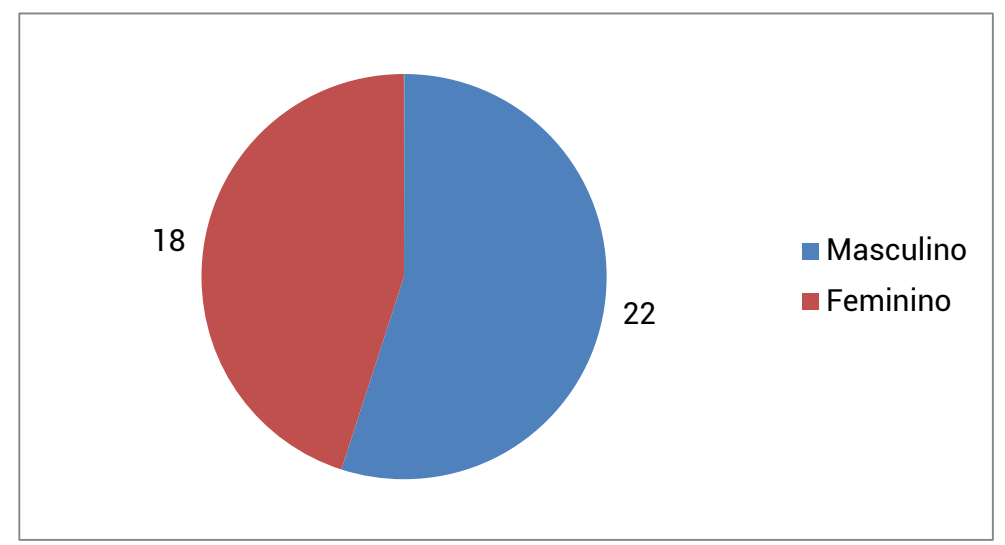

Fonte: Pesquisa do autor

A faixa etária (Gráfico 2) dividiu-se em $n=22$ idosos (55\%) entre 60 e 70 anos; $n=11$ idosos (27,5\%) entre 71 e 80 anos; $n=5$ idosos (12,5\%) entre 81 e 90 anos e $n=2$ idosos (5\%) maior de 90 anos. Com relação à idade, sabe-se que, à medida que o ser humano envelhece, aquelas tarefas cotidianas consideradas banais vão paulatinamente e, muitas vezes, de forma imperceptível, tornando-se de difícil realização até o momento em que o indivíduo nota ser necessário auxílio de outrem para execução delas (ARAÚJO; CEOLIM, 2007). Segundo Guccione (2002), a capacidade de funcionar de modo independente declina com a idade e esse declínio é influenciado por um conjunto de fatores biológicos, psicológicos e sociais.

\section{Gráfico 2 - Distribuição de faixa etária}

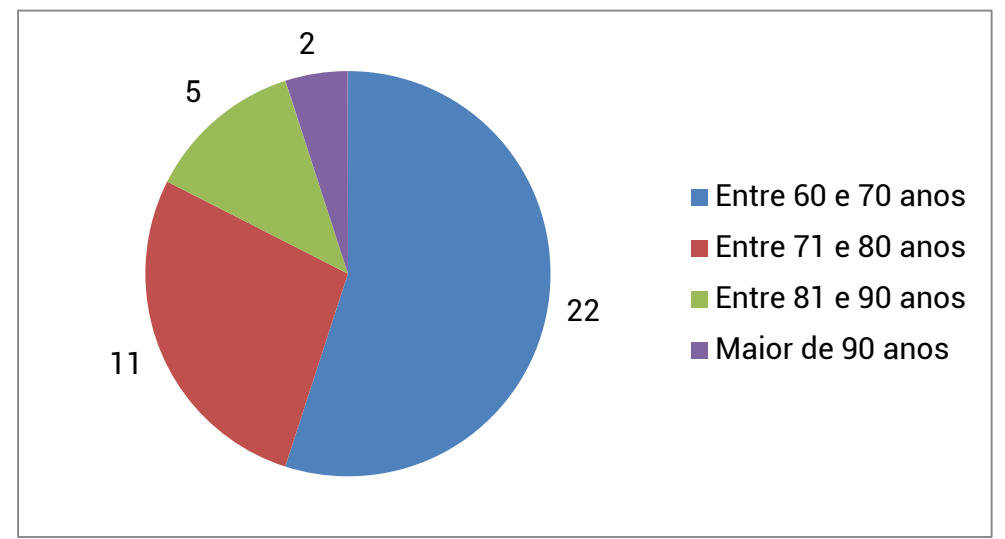

Fonte: Pesquisa do autor

$\mathrm{Na}$ capacidade funcional (Gráfico 3), $\mathrm{n}=31$ idosos $(77,5 \%)$ foram classificados com nenhuma ou com alteração leve da capacidade funcional; $n=8(20 \%)$ idosos, com alteração moderada da capacidade funcional e $n=1$ idoso (2,5\%), com alteração grave da capacidade funcional. Como notado, quase $80 \%$ dos participantes possuíam uma boa capacidade funcional. A Política Nacional de Saúde do Idoso assume que o principal problema que pode afetar o idoso, como consequência da evolução de suas enfermidades e de seu estilo de vida, é a perda de sua capacidade funcional, isto é, a perda das habilidades físicas e mentais necessárias para a realização de suas atividades básicas e instrumentais da vida diária (SILVESTRE, 2003). Alves et al. (2007) pontuam que a manutenção da capacidade funcional pode 
ter implicações para a qualidade de vida dos idosos, pois está diretamente relacionada com a forma pela qual o indivíduo se mantém na comunidade, desfrutando a sua independência até as idades mais avançadas. Nesse sentido, a Estratégia de Saúde da Família atua com papel fundamental ao contribuir planejando programas preventivos para detecção precoce e a eliminação de fatores de risco relacionados à incapacidade. É importante comentar que saúde não é apenas uma questão de assistência médica e de acesso a medicamentos, mas sim a promoção de "estilos de vida saudáveis", encarada pelo sistema de saúde como uma ação estratégica (BRASIL, 2009). A unidade de saúde em questão possui grupos de idosos, onde há encontros semanais para apresentação de temas relacionados à saúde e o bem-estar dos usuários. Além disso, os idosos participam da "Academia da Terceira Idade", programa de cunho municipal, onde realizam atividade física semanal ao "ar livre", com orientação de educadores físicos.

Gráfico 3 - Comprometimento da capacidade funcional

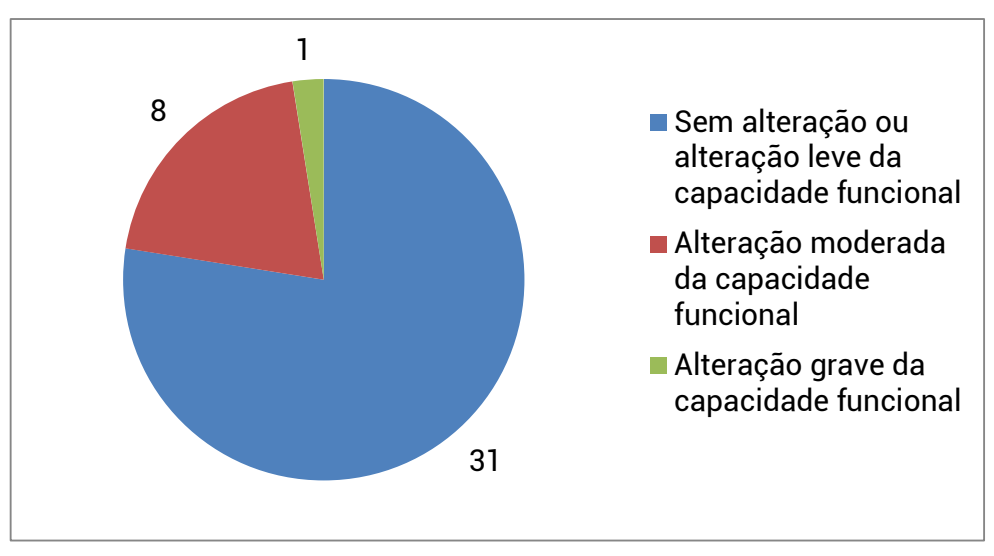

Fonte: Pesquisa do autor

Em relação à variável Gênero x capacidade funcional (Gráfico 4), $n=17$ idosos (42,5\%) do sexo masculino e $n=14$ idosos (35\%) do sexo feminino foram classificados com nenhuma ou alteração leve da capacidade funcional, totalizando os $77,5 \%$ dos participantes, como mencionado previamente. Com alteração moderada da capacidade funcional, $n=4$ idosos (10\%) do sexo masculino e $n=4(10 \%)$ do sexo feminino. E com alteração grave da capacidade funcional apenas um participante do sexo masculino, $n=1$ idoso $(2,5 \%)$.

Gráfico 4 - Relação entre Gênero x Capacidade Funcional

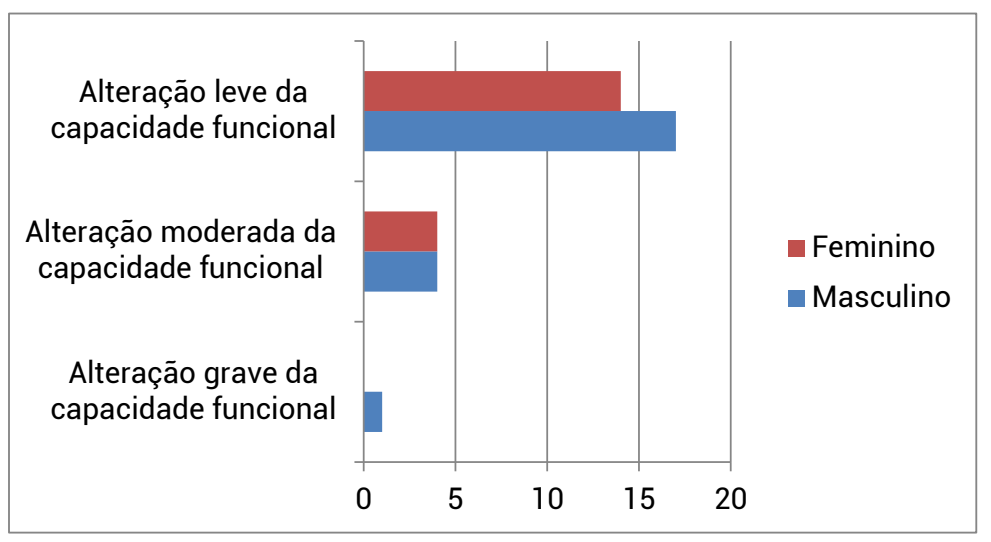

Fonte: Pesquisa do autor 
Analisando a variável Faixa etária x capacidade funcional (Gráfico 5), dos 22 participantes com a faixa etária entre 60 e 70 anos, todos os entrevistados (100\%) possuíam nenhuma ou uma alteração leve da capacidade funcional. Um resultado bem expressivo e que reflete diretamente na qualidade de vida desses idosos, pois mostra que o entrevistado tem condições funcionais suficientes para viver de forma autônoma e independente, mantendo suas atividades cotidianas, como tomar banho, vestirse, realizar higiene pessoal, transferir-se, alimentar-se, manter a continência, preparar refeições, ter o controle financeiro, etc.

Dos que tinham entre 71 e 80 anos, $n=8$ idosos (20\%) possuíam nenhuma ou uma alteração leve da capacidade funcional e $\mathrm{n}=3$ idosos $(7,5 \%)$ apresentaram alteração moderada da capacidade funcional. Esse número é bastante relevante, um pouco mais de $70 \%$ dos entrevistados nessa faixa etária possuíam uma boa capacidade funcional.

Entre 81 e 90 anos, do total de 5 participantes, $n=1$ idoso (2,5\%) não possuía ou possuía alteração leve da capacidade funcional e $n=4$ idosos $(10 \%)$ apresentaram alteração moderada da capacidade funcional. Dos dois entrevistados que eram maiores de 90 anos, $n=1$ idoso $(2,5 \%)$ possuía alteração moderada da capacidade funcional e $\mathrm{n}=1$ idoso $(2,5 \%)$ apresentou alteração grave da capacidade funcional. Os últimos resultados refletem diretamente a citação anterior, ao afirmar que, com o avançar da idade, naturalmente, há um decréscimo já esperado da capacidade funcional.

\section{Gráfico 5 - Relação entre Faixa Etária x Capacidade Funcional}

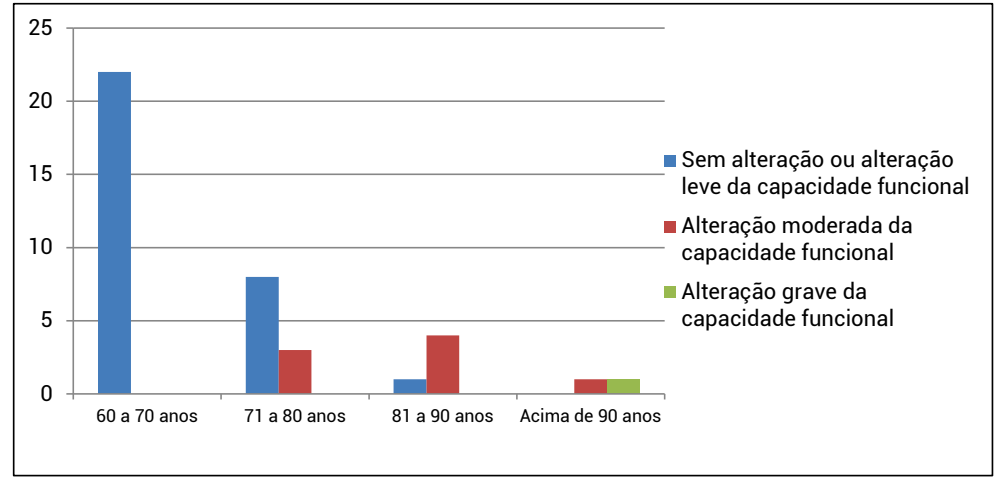

Fonte: Pesquisa do autor

\section{CONCLUSÃO}

Diante dos resultados obtidos, notou-se que quase $80 \%$ dos participantes apresentaram uma boa capacidade funcional, sendo classificados em "sem incapacidade" ou com "alteração leve da capacidade funcional". A manutenção da capacidade funcional possui um papel fundamental na qualidade de vida dos idosos por estar relacionada com a possibilidade de manter um estilo de vida saudável, uma função no ambiente familiar e social em que vive e de estar inserido em uma rede social. Alves (2007) afirma que, quando o idoso está comprometido funcionalmente, a família é afetada também, já que a incapacidade ocasiona maior vulnerabilidade e dependência na velhice, contribuindo para a diminuição do bem-estar e da qualidade de vida dos idosos.

Nesse sentido, a Política Nacional de Saúde do Idoso apresenta 
como propósito basilar a promoção do envelhecimento saudável, a manutenção e a melhoria, ao máximo, da capacidade funcional dos idosos, a prevenção de doenças, a recuperação da saúde dos que adoecem e a reabilitação daqueles que venham a ter a sua capacidade funcional restringida, de modo a garantir-lhes permanência no meio em que vivem, exercendo de forma independente suas funções na sociedade. (BRASIL, 1999, p. 21)

Assim, a estratégia de Saúde da Família tem seu papel fundamental ao fornecer à população idosa, através do Médico da Família, ações de promoção de saúde, detecção e intervenção precoce de doenças e reabilitação, diminuindo os fatores de risco e evitando a incapacidade funcional. Além disso, permite uma interação maior com a família do paciente idoso, pois conhece as suas peculiaridades, fornecendo, portanto, ajuda de maneira mais eficiente.

\section{REFERÊNCIAS}

ALENCAR, M. C. B.; HENEMANN, L.; ROTHENBUHELER, R. A capacidade funcional de pacientes, e a fisioterapia em um programa de assistência domiciliar. Revista Fisioterapia em Movimento, v. 21, n. 1, p. 11-20, 2008.

ALVES, L. C.; LEIMANN, B. C. Q.; VASCONCELOS, M. E. L.; CARVALHO, M. S.; VASCONCELOS, A. G. G.; FONSECA, T. C. O.; LEBRÃO, M. L.; LAURENTI, R. A influência das doenças crônicas na capacidade funcional dos idosos do município de São Paulo, Brasil. Cad. Saúde Pública. V. 23, n. 8, p. 1924-30, ago., 2007.

BRASIL, 1999. Portaria do Gabinete do Ministro de Estado da Saúde de $n^{\circ} 1395$, de 9 de dezembro de 1999, que aprova a Política Nacional de Saúde do Idoso e dá outras providências. Brasília: Diário Oficial (da) República Federativa do Brasil, nº 237-E, pp.20-24, 13 dez., seção 1.

BRASIL. Ministério da Saúde. Saúde do Idoso. Portal da Saúde, 2009. Disponível em: <http://portal. saude.gov.br/ portal/saude/visualizar_texto.cfm?idtxt=26466>. Acesso em: 13 ago. 2012.

BRASIL. Ministério da Saúde. Secretaria de Atenção à Saúde. Departamento de Atenção Básica. Envelhecimento e saúde da pessoa idosa / Ministério da Saúde, Secretaria de Atenção à Saúde, Departamento de Atenção Básica, Brasília: Ministério da Saúde, 2006.

BRUCE, B.; FRIES, J. F. The stanford health assessment questionnaire: a review of its history, issues, progress, and documentation. The Journal of Rheumatology, v. 30, n. 1, p. 167-78, 2003. Disponível em: <http://aramis.stanford. edu/downloads/JRheumatol_new\%20HAQ.pdf>. Acesso em: 13 ago. 2012.

COSTA, E. C.; NAKATANI, A. Y. K.; BACHION, M. M. Capacidade de idosos da comunidade para desenvolver Atividades de Vida Diária e Atividades Instrumentais de Vida Diária. Acta Paul. Enferm. V. 19, n.1, p. 43-8, 2006.

COSTA, E.F.A.; Porto, C.C.; Almeida, J.C. et al. Semiologia do idoso. In: Porto, C.C. (ed). Semiologia Médica. 4. ed. Rio de Janeiro: Guanabara Koogan, 2001. (9):165-197.

DUARTE, Y. A. O.; ANDRADE, C. L A.; LEBRÃO, M L. 0 Índice de Katz na avaliação da funcionalidade dos idosos. Revista da Escola de Enfermagem - USP, v. 41, n. 2, p. 317-25, 2007.

GUCCIONE, A. A. Fisioterapia geriátrica. 2. Ed. Rio de Janeiro: Guanabara Koogan, 2002. 
LACOURT, M. X.; MARINI, L. L. Decréscimo da função muscular decorrente do envelhecimento e a influência na qualidade de vida do idoso: uma revisão de literatura. RBCEH - Revista Brasileira de Ciências do Envelhecimento Humano, v. 3, n. 1, p. 114-121, 2006.

NAKATANI, A. Y. K. et al. Perfil sócio-demográfico e avaliação funcional de idosos atendidos por uma equipe de saúde da família na periferia de Goiânia. Revista da Sociedade Brasileira de Clínica Médica, v. 5, n. 1, p. $131-136,2003$.

PACHECO, R. O.; SANTOS, S. S.C. Avaliação global de idosos em unidades de PSF. Textos sobre Envelhecimento. v. 7, n. 2, 2004.

PAPALÉO NETTO, M. Tratado de gerontologia. 2. ed. rev. ampl. São Paulo (SP): Ed. Atheneu; 2007. p.39-56.

ROSA, T. E. C. et al. Fatores determinantes da capacidade funcional entre idosos. Revista de Saúde Pública, v. 37, n. 1, p. 40-8, 2003.

SANTANA SCHNEIDER, R. H.; MARCOLIN, D.; DALACORTE, R. R. Avaliação funcional de idosos. Revista S. Médica, v. 18, n. 1, p. 4-9, 2008.

SILVESTRE J.A.; Costa Neto, M.M. Abordagem do idoso em programas de saúde da família. Cad. Saúde Pública, Rio de Janeiro, 19(3):839-847, mai-jun, 2003.

VERAS, R. Envelhecimento populacional contemporâneo: demandas, desafios e inovações. Revista de Saúde Pública. v 43, n. 3, p. 548-54, 2009. 